\title{
High-resolution ultrasonography in evaluating peripheral nerve entrapment and trauma
}

\author{
Ralph W. Koenig, M.D., Maria T. Pedro, Christian P. G. Heinen, M.D., \\ Thomas Schmidt, Hans-Peter Richter, M.D., Ph.D., Gregor Antoniadis, M.D., Ph.D., \\ and Thomas Kretschmer, M.D., Ph.D.
}

Department of Neurosurgery, University of Ulm, Germany

\begin{abstract}
High-resolution ultrasonography is a noninvasive, readily applicable imaging modality, capable of depicting real-time static and dynamic morphological information concerning the peripheral nerves and their surrounding tissues. Continuous progress in ultrasonographic technology results in highly improved spatial and contrast resolution. Therefore, nerve imaging is possible to a fascicular level, and most peripheral nerves can now be depicted along their entire anatomical course. An increasing number of publications have evaluated the role of high-resolution ultrasonography in peripheral nerve diseases, especially in peripheral nerve entrapment.

Ultrasonography has been shown to be a precious complementary tool for assessing peripheral nerve lesions with respect to their exact location, course, continuity, and extent in traumatic nerve lesions, and for assessing nerve entrapment and tumors. In this article, the authors discuss the basic technical considerations for using ultrasoniography in peripheral nerve assessment, and some of the clinical applications are illustrated. (DOI: $10.3171 /$ FOC.2009.26.2.E13)
\end{abstract}

KEY WoRds $\bullet \quad$ entrapment $\quad$ high-resolution ultrasonography $\bullet$
peripheral nerves $\quad \bullet \quad$ traumatic nerve lesions

$\mathrm{S}$ INCE the earliest reports of peripheral nerve ultrasonography by Solbiati et al..$^{38}$ and Fornage ${ }^{15}$ technical progress in ultrasonography has been ongoing. Aside from neurophysiological assessment of peripheral nerve lesions, the technique of high-resolution ultrasonography provides an increasing amount of complementary morphological information about nerves and their surrounding tissues. High-resolution ultrasonography images depict nerves in high image quality and enable clinicians to examine them along their anatomical course, thus giving valuable information about the origin and exact location of most lesions. ${ }^{13,28}$ Additionally, ultrasonography provides the possibility of dynamic imaging of peripheral nerves.

In the present study, we review the current state of high-resolution ultrasonography for imaging peripheral nerve entrapment and traumatic peripheral nerve lesions with case illustrations.

\section{Technical Considerations}

Ultrasonography scanners of the latest generation can depict subtle details of the peripheral nerves. This

\footnotetext{
Abbreviations used in this paper: CuTS = cubital tunnel syndrome; CTS = carpal tunnel syndrome.
}

marks the temporary end point in the continuous and ongoing improvement of ultrasonographic technology: high-resolution transducers of up to $18 \mathrm{MHz}$ have been developed that enable an axial resolution of up to 400 $\mu \mathrm{m}$. In parallel to those transducers, scanner software (such as compound imaging, ,36,19,25 tissue harmonic imaging, ${ }^{35}$ and high-resolution imaging) was developed, further improving image quality by reducing artifact and increasing contrast and spatial resolution.

With higher ultrasonographic frequencies there is less penetration of deep tissues; therefore, the choice of transducers very much depends on the nerve and anatomic region under examination. In general, the highest frequency possible should be used for examination. Superficially located nerves such as the median nerve should be examined with transducers of 15-18 MHz, whereas deep nerves such as the sciatic nerve or the brachial plexus are better examined with 9-12 MHz transducers.

\section{Ultrasonography of Normal Peripheral Nerves}

Fornage $^{15}$ was the first to systematically examine the appearance of peripheral nerves under high-resolution ultrasonography. Silvestri and colleagues ${ }^{37}$ subsequently described the normal internal architecture of peripheral nerves on longitudinal sections as multiple hypoechoic 
parallel but discontinuous linear areas separated by hyperechoic bands. On transverse sections multiple round hypoechoic areas were seen on a homogeneous hyperechoic background. Side-by-side comparison between transverse ultrasonography scans and histological studies demonstrated, that the hypoechoic areas on ultrasonography correspond to fascicles of neuronal fibers, the hyperechoic background to the epineurium. However, the number of fascicles seen under light microscopy was higher than that on ultrasonography; thus, ultrasonography underestimated the number of fascicles.

Silvestri et al. ${ }^{37}$ emphasizes the correct differentiation between tendons, ligaments, and nerves, which may be difficult for beginners on nerve sonograms. Compared to tendons, nerves show differences in echotexture, and tendons tend to be more hyperechoic. Silvestri and colleagues differentiated between a "fibrillar echotexture" of tendons and a "fascicular nerve echotexture." Dynamic maneuvers like passive movement of the fingers or joints turn out to be helpful in differentiating between mobile tendons and comparatively static nerves during scanning.

Orientation during ultrasonographic examination of the peripheral nerves is further facilitated by the identification of specific anatomical landmarks such as the scaphoid bone in the carpal tunnel or the deep radial artery lying next to the radial nerve in the upper arm.

\section{Peripheral Nerve Entrapment}

Recently, several sites of nerve entrapment in the upper as well as lower extremity amenable to ultrasonographic examination were described. . $^{8} 12,18,26,34,29,30$ Many authors have dealt explicitly with the use of ultrasonography in carpal tunnel ${ }^{5,10,14,21,23,24,45}$ and cubital tunnel syndrome. ${ }^{3,4,29}$ Regardless of the different entrapment sites, peripheral nerves show typical uniform alterations in compression syndromes: fusiform swelling proximal to the compression site ("pseudoneuroma"), caused primarily by intraneural venous congestion and edema ${ }^{39}$ and frequently followed by a sudden decrease in caliber at the ultimate point of compression. ${ }^{29}$ The echotexture of entraped nerves is altered, hypoechoic, and the physiological fascicular echotexture appears rarefied or completely lost. Doppler imaging studies frequently reveal increased flow patterns reflecting intraneural hyperemia. ${ }^{27}$

Quantitative assessment of nerve thickening performed by measuring the maximum cross-sectional area seems to be the most reliable method for the diagnosis of nerve entrapment. ${ }^{2}$ There are many discrepancies among the different studies, and many different criteria are used in the diagnosis. In some cases the criteria used are not adequately described. For example, most studies do not report whether the hyperechoic rim that encompasses the nerve was included in measurements of the nerve's cross-sectional area. ${ }^{31}$ Apart from the known interindividual differences in nerve thickness, there may be a cause for thebroad range of normative values reported in the literature. ${ }^{17,40-42}$

\section{Carpal Tunnel Syndrome}

In 1991 Buchberger et al..$^{10}$ described the diagnostic

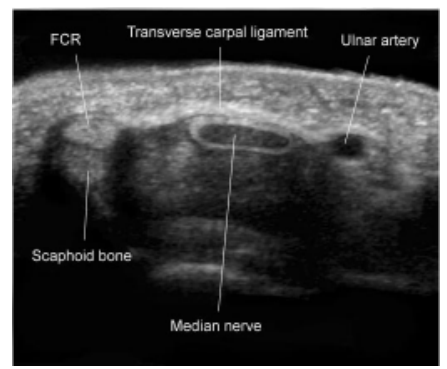

Fig. 1. Transverse sonogram of the median nerve at the level of the proximal carpal tunnel showing a slightly enlarged nerve cross-sectional area and bowing of the transverse carpal ligament. FCR = tendon of flexor carpi radial muscle.

triad for CTS on ultrasonography (Fig. 1): proximal median nerve swelling, bowing of the flexor retinaculum, and distal nerve flattening. Since then, the usefulness and reliability of various ultrasonographic criteria and diagnostic algorithms for CTS have been discussed in detail. $5,9,11,14,31,43$ In the end, enlargement of median nerve cross-sectional area at the proximal level of the carpal tunnel is the most frequently used criterion for the ultrasonographic diagnosis of CTS. ${ }^{1}$ Nevertheless the critical threshold of the median nerve cross-sectional area differs considerably among various studies, and the limiting value indicating CTS ranges from 9 to $15 \mathrm{~mm}^{2} .^{17,41,42}$ Hobson-Webb and coworkers ${ }^{20}$ proposed the use of an ultrasonographic wrist-to-forearm median nerve area ratio in the diagnosis of CTS. This ratio takes into account the normal variation in median nerve thickness. Using a wrist-to-forearm median nerve area ratio of $\geq 1.4$ resulted in a $100 \%$ sensitivity for detecting CTS, and was a superior method to measuring the median nerve cross-sectional area at the proximal carpal tunnel only. Subsidiary local disturbances in intraneural and perineural microvascularity, especially hyperemia, detected on Doppler ultrasonography seem to predict median nerve entrapment. ${ }^{27}$

As a tool for morphological examination, ultrasonography is capable of differentiating idiopathic from secondary CTS. Various abnormalities in the tunnel such as ganglia, tumors, flexor tendon synovitis, or accessory muscle bellies can be detected easily. Persistent median arteries, frequently associated with anatomic variations in the median nerve itself, such as a bifid median nerve or high nerve division, can be found in up to $26 \%$ of cases. ${ }^{16}$

Overall CTS remains a clinical diagnosis. Nevertheless, ultrasonography is useful in selected cases because it can detect anatomical variations and the causes of secondary CTS, such as tumors or ganglia.

Ultrasonography is especially useful in evaluating patients who do not show improvement after carpal tunnel release. Iatrogenic nerve injuries and nontransected or partially transected, retinacula can be depicted. Various other causes for ongoing nerve entrapment such as fluid collections (hematoma or abscess) or scar tissue encasing the nerve can also be detected.

\section{Cubital Tunnel Syndrome}

Like in other entrapment syndromes the main ultra- 


\section{High-resolution ultrasonography and peripheral nerve entrapment}

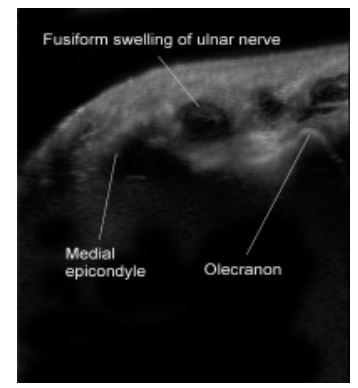

FIG. 2. Transverse sonogram of the ulnar nerve at the level of the medial epicondyle (with the elbow extended) showing marked swelling of the ulnar nerve proximal to the cubital tunnel in CuTS.

sonographic sign of CuTS is focal nerve swelling with loss of fascicular pattern ${ }^{29}$ just proximal to the entrapment site (Fig. 2). Anatomical variations of the surrounding anatomy and tissues, such as bone prominences or flattening of the medial epicondyle, ganglia, or an overriding triceps tendon, can be detected easily and may be valuable in the preoperative workup in patients with CuTS.

Ultrasonographic measurement of the ulnar nerve at elbow level depends on various individual factors such as patient age, weight, sex, and elbow position..$^{40}$ Because of these interindividual differences similar to CTS, ${ }^{20}$ Yoon and colleagues ${ }^{44}$ proposed the use of a ratio between the cross-sectional area at the site of maximal enlargement and at an unaffected site of the nerve, such as the middle of the upper arm to improve diagnostic accuracy. A cutoff ratio of 1.5 was chosen; 1 yielded a sensitivity of $100 \%$ and specificity of $96.7 \%$ for the diagnosis of CuTS.

Real-time dynamic sonographic imaging during flexion and extension is very useful especially in the diagnostic workup for CuTS. Okamoto et al. ${ }^{32}$ classified 3 types of nerve dislocation during movement: Type $\mathrm{N}$ (no dislocation), Type S (subluxation), and Type D (dislocation). In various studies, Types $\mathrm{S}$ and $\mathrm{D}$ dislocation have been confirmed with a frequency of 24.3-47\%.22,32 At least in our department dislocation of the ulnar nerve during movement directly influences surgical decompression (simple decompression versus anterior submuscular transposition for ulnar nerve dislocation).

Ultrasonography imaging is also useful in outlining the ulnar nerve's course after those unrewarding revision surgery cases in which the nerve's course over the elbow

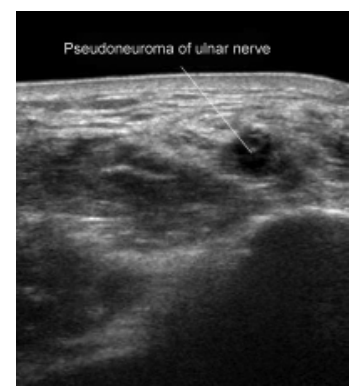

FIG. 3. Case 1. Transverse sonogram of the ulnar nerve after subcutaneous anterior transposition at the proximal forearm level (with the elbow extended) showing marked swelling of the ulnar nerve proximal to the cubital tunnel in CuTS. is completely unclear. Nerve kinking, scar tissue, or insufficient transposition with the nerve riding on the medial epicondyle can easily be detected, and the nerve's course can be outlined preoperatively on the patient's skin.

\section{Illustrative Case 1}

This 45-year-old man suffered from persistent paresis and dysesthesia after primary decompression and revision surgery for CuTS at an outside institution. Recordings of these procedures were not available. On examination, the nerve could not be palpated inside the sulcus, and the 6-cm-long scar was in the typical location and looked inconspicuous. Electromyography revealed marked signs of chronic denervation.

On ultrasonography the nerve was obviously transposed and could be tracked subcutaneously (Fig. 3). The surrounding tissue was scared and precondylar kinking due to preserved intermuscular septum was detected.

At revision surgery, microsurgical neurolysis of the ulnar nerve with resection of the intermuscular septum and deep submuscular anterior transposition of the nerve was performed, resulting in good results with improvement of symptoms.

\section{Illustrative Case 2}

This 72-year-old man presented with a tardy ulnar nerve palsy related to an elbow fracture sustained 10 years ago. On examination there was advanced atrophy of the intrinsic hand muscles and marked hypesthesia of ulnar nerve distribution.

Ultrasonographic images revealed a deformed elbow joint, an apparently normal proximal ulnar nerve, marked swelling of the nerve a short distance before the sulcus, and hypoechogeneity. No fascicles could be depicted within the nerve (Fig. 4). In the sulcus itself there was a sudden flattening of the nerve caused by an underlying elbow deformation.

External neurolysis and deep submuscular anterior transposition were performed, and the ultrasonographic findings were confirmed intraoperatively.

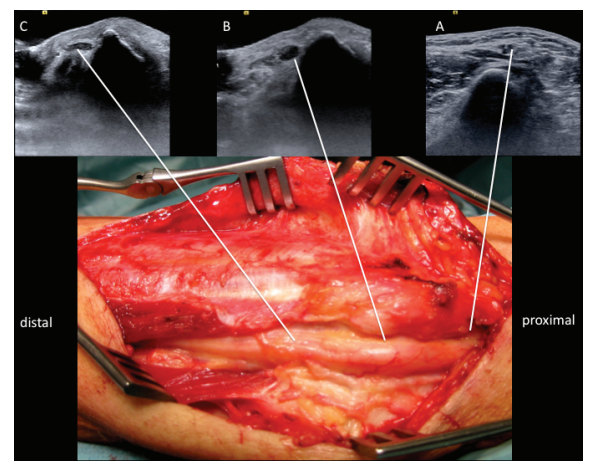

FIG. 4. Case 2. Intraoperative image and corresponding transverse sonograms. Normal ulnar nerve with fascicular pattern proximal to the ulnar sulcus (A), significant swelling of the ulnar nerve in the sulcus ulnaris with loss of nerve echotexture $(B)$, and flattening of the ulnar nerve due to underlying joint deformation $(\mathrm{C})$ are shown. 


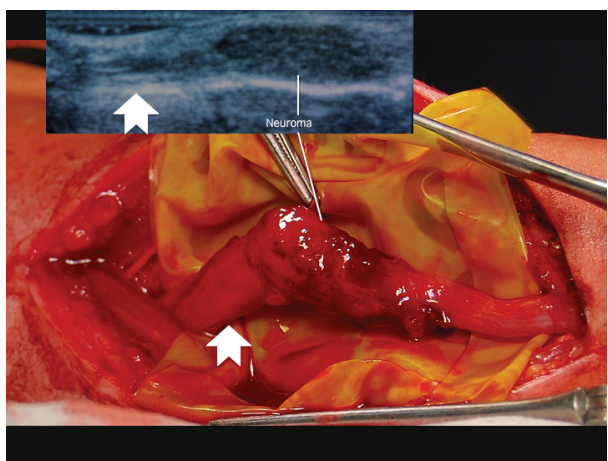

FIG. 5. Case 3. Intraoperative image and corresponding longitudinal sonogram showing neuroma of ulnar nerve after injury with a piece of broken glass. The arrow marks the intact part of ulnar nerve, proximal to the lesion with fascicular echotexture (sonogram taken with an older scanner, 12-MHz transducer, note the marked difference in quality compared to the other figures).

\section{Traumatic Peripheral Nerve Lesions}

Supplementary evaluation of traumatic nerve lesions with ultrasonography will soon gain more importance. Morphological examination of the peripheral nerves in the context of traumatic injury is extremely useful, especially in closed injuries. In addition to preoperative clinical and electrophysiological examinations, high-resolution ultrasonography can contribute substantially to the appropriate management of traumatic peripheral nerve lesions. ${ }^{6,7,33}$

In their small study, Bodner and colleagues ${ }^{6}$ were able to demonstate that severe nerve damage (such as laceration or loss of continuity) and impairment of the nerve by surrounding posttraumatic tissue such as callus, bone fragments, or scarring can reliably be visualized on ultrasonography. Consequently, in nerves amenable to ultrasonographic examination, differentiation between severe damage needing surgical repair and milder lesions is possible. This might lead to earlier surgical intervention, with improved outcomes.

However, criteria for ultrasonographic interpretation of nerve lesions in continuity are not yet clearly defined. At present high-resolution ultrasonography is the only imaging modality that enables ultrastructural examination of the peripheral nerves. It may be possible to define the morphological ultrasonography criteria that reveal the extent and depth of traumatic nerve lesions in continuity. Therefore, intraoperative findings and intraoperative compound nerve action potential measurements must be correlated with preoperative sonograms to obtain a thorough understanding of this modality's capability to reflect a complete neuroma in continuity. A provisional-but reasonable-assumption would be that the chances of spontaneous recovery are low when the fascicular structure is seen proximal and distal to a lesion, but not within it.

Another useful aspect of ultrasonography is the possibity to detect proximal and distal nerve stumps, which can be marked on the skin preoperatively, such as in accessory nerve lesions. ${ }^{7}$

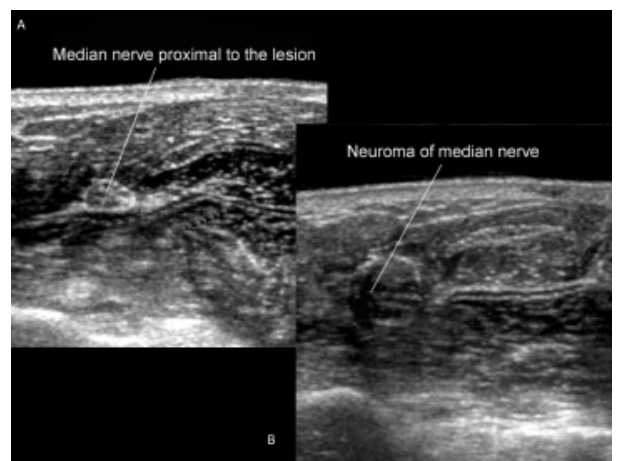

FIG. 6. Case 4. A: Transverse sonogram of the median nerve proximal to the lesion showing the nerve with preserved fascicular pattern. B: Transverse sonogram of the median nerve $2-3 \mathrm{~cm}$ distal showing neuroma-in-continuity. Note hypoechogeneity, loss of fascicular echotexture, and marked swelling.

\section{Illustrative Case 3}

This 28-year-old man presented to our institution 2 months after a piece of broken glass cut his proximal ulnar forearm. The wound had been cleaned and sutured with a few stitches at a local hospital and nerve damage was not reported during wound exploration. On presentation the patient exhibited complete ulnar nerve palsy.

A sonogram obtained with an old scanner $(12-\mathrm{MHz}$ transducer; no tissue harmonic imaging or compound imaging) revealed a complete neuromatous nerve lesion (Fig. 5).

A complete traumatic lesion of the ulnar nerve was confirmed intraoperatively, and microsurgical nerve grafting with $4 \times 4-\mathrm{cm}$ sural transplant grafts was necessary.

\section{Illustrative Case 4}

This 59-year-old woman presented to our institution 3 months after a motor saw injury to the distal right forearm. She suffered from severe soft tissue traumatic injuries with complete transsection of the radial artery, various tendons, and the median nerve. Immediately after trauma the radial artery and several tendons were reconstructed during the primary surgery; according to the operative report of the local trauma center the median nerve was primarily reconstructed. On presentation the patient complained of neuropathic pain, and her skin appeared glassy and edematous. Examination revealed a complete motor and sensory deficit of the median nerve distribution.

Ultrasonographic imaging showed the nerve to be in continuity (Fig. 6). Proximal and distal to the nerve there was the typical inconspicuous fascicular pattern interrupted by $\mathrm{a} \sim 4$-cm-long intersection of nerve that was markedly swollen, hypoechogeneic, and lacking fascicles.

Based on these findings, we proposed early revision surgery, but the patient was not willing to undergo another operation at that time. 


\section{High-resolution ultrasonography and peripheral nerve entrapment}

\section{Conclusions}

Because of the continuous and speedy development of ultrasound technology, current ultrasound scanners together with high frequency linear array transducers can depict nerves with a spatial resolution as high as $400 \mu \mathrm{m}$ (axial resolution). Consequently, most peripheral nerves can be scanned in their anatomical course and their fascicular pattern can be analyzed.

The primary focus of the published literature is the ultrasonographic appearance of normal peripheral nerves and the definition and evaluation of ultrasonographic criteria for nerve entrapment.

Although high-resolution ultrasonography is a helpful way to evaluate nerve entrapment, in our view it is even more important in the assessment of peripheral nerve trauma, tumors, and in conducting preoperative evaluations before revision surgeries. Ultrasonography can depict nerves in their anatomical or altered courses, giving the surgeon a clear idea of the intraoperative problems he will face.

Despite the fact that the role of ultrasonography in traumatic nerve lesions is not yet clearly defined, together with clinical and electrophysiological examinations, it may be able to better delineate the extent and depth of a lesion. In cases of nerve disruptions, surgical decision making, and management will be straightforward. Moreover, it might be possible to define ultrasonographic criteria for evaluating lesions in continuity. This information could enable surgeons to evaluate the potential for spontaneous recovery, implying also that candidates for revision surgery could be identified earlier in their course. Preoperative ultrasonography images (for examining echotexture, echogeneity, and quality of surrounding tissue) must therefore be correlated with intraoperative findings and the results of intraoperative compound nerve action potential measurements and the histological characteristics of biopsy samples.

\section{Acknowledgment} figures.

The authors acknowledge Markus Schönl for preparing the

\section{Disclaimer}

The authors report no conflict of interest concerning the materials or methods used in this study or the findings specified in this paper.

\section{References}

1. Alemán L, Berná J, Reus M, Martínez F, Donénech-Ratto G, Campos M: Reliability of sonographic measurements of the median nerve. J Ultrasound Med 27:193-197, 2008

2. Bargfrede M, Schwennicke A, Tumani H, Reimers CD: Quantitative ultrasonography in focal neuropathies as compared to clinical and EMG findings. Eur J Ultrasound 10:21-29, 1999

3. Beekman R, Schoemaker MC, Van der Plas JP, Van den Berg LH, Franssen H, Wokke JH, et al: Diagnostic value of highresolution sonography in ulnar neuropathy at the elbow. Neurology 62:767-773, 2004

4. Beekman R, Van der Plas JP, Uitdehaag BM, Schellens RL,
Visser LH: Clinical, electrodiagnostic and sonographic studies in ulnar neuropathy at the elbow. Muscle Nerve 30:202208, 2004

5. Bianchi S, Montet X, Martinoli C, Bonvin F, Fasel J: High-resolution sonography of compressive neuropathies of the wrist. J Clin Ultrasound 32:451-461, 2004

6. Bodner G, Buchberger W, Schocke R, Bale R, Huber B, Harpf $C$, et al: Radial nerve palsy associated with humeral shaft fracture: evaluation with US-initial experience. Radiology 219:811-816, 2001

7. Bodner G, Harpf H, Gardetto A, Kovacs P, Gruber H, Peer S, et al: Ultrasonography of the accessory nerve. Normal and pathologic findings in cadavers and patients with iatrogenic accessory nerve palsy. J Ultrasound Med 21:1159-1163, 2002

8. Bodner G, Harpf C, Meirer R, Gardetto A, Kovacs P, Gruber $\mathrm{H}$ : Ultrasonographic appearance of supinator syndrome. J UItrasound Med 21:1289-1293, 2002

9. Buchberger W, Judmaier W, Birbamer G, Lener M, Schmidauer C: Carpal tunnel syndrome: diagnosis with high-resolution sonography. AJR Am J Roentgenol 159:793-798, 1992

10. Buchberger W, Schön G, Strasser K, Jungwirth W: High-resolution ultrasonography of the carpal tunnel. J Ultrasound Med 10:531-537, 1991

11. Chen P, Maklad N, Redwine M, Zelitt D: Dynamic high-resolution sonography of the carpal tunnel. AJR Am J Roentgenol 168:533-537, 1997

12. Chien AJ, Jamadar DA, Jacobson JA, Hayes CW, Louis DS: Sonography and MR imaging of posterior interosseous nerve syndrome with surgical correlation. AJR Am J Roentgenol 181:219-221, 2003

13. Chiou HJ, Chou YH, Chiou SY, Liu JB, Chang CY: Peripheral nerve lesions: role of high-resolution US. Radiographics 23:E15, 2003

14. Duncan I, Sullivan P, Lomas F: Sonography in the diagnosis of carpal tunnel syndrome. AJR Am J Roentgenol 173:681684, 1999

15. Fornage BD: Peripheral nerves of the extremities: imaging with US. Radiology 167:179-182, 1988

16. Gassner EM, Schocke M, Peer S, Schwabegger A, Jaschke W, Bodner G: Persistent median artery in the carpal tunnel. Color doppler ultrasonographic findings. J Ultrasound Med 21:455-461, 2002

17. Hammer HB, Hovden IAH, Haavardsholm EA, Kvien TK: Ultrasonography shows increased cross-sectional area of the median nerve in patients with arthritis and carpal tunnel syndrome. Rheumatology 45:584-588, 2006

18. Hide IG, Grainger AJ, Naisby GP, Campbell RS: Sonographic findings in the anterior interosseous nerve syndrome. J Clin Ultrasound 27:459-464, 1999

19. Jespersen SK, Wilhjelm JE, Sillesen H: Multi-angle compound imaging. Ultrason Imaging 20:81-102, 1998

20. Hobson-Webb LD, Massey JM, Juel VC, Sanders DB: The ultrasonographic wrist-to-forearm median nerve area ratio in carpal tunnel syndrome. Clin Neurophysiol 119:1353-1357, 2008

21. Kele H, Verheggen R, Bittermann HJ, Reimers CD: The potential value of ultrasonography in the evaluation of carpal tunnel syndrome. Neurology 61:389-391, 2003

22. Kim BJ, Date ES, Lee SH, Yoon JS, Hur SY, Kim SJ: Distance measure error induced by displacement of the ulnar nerve when the elbow is flexed. Arch Phys Med Rehabil 86:809812,2005

23. Kotevoglu N, Gulbahce-Saglam S: Ultrasound imaging in the diagnosis of carpal tunnel syndrome and its relevance to clinical evaluation. Joint Bone Spine 72:142-145, 2005

24. Koyuncuoglu HR, Kutluhan S, Yesildag A, Oyar O, Guler K, Özden A: The value of ultrasonographic measurement in carpal tunnel syndrome in patients with negative electrodiagnostic tests. Eur J Radiol 56:365-369, 2005 
25. Lin DC, Nazarian LN, O'Kane PL, McShane JM, Parker L, Merritt CR: Advantages of real-time spatial compound sonography of the musculoskeletal system versus conventional sonography. AJR Am J Roentgenol 179:1629-1631, 2002

26. Lee D, Dauphinée DM: Morphological and functional changes in the diabetic peripheral nerve: using diagnostic ultrasound and neurosensory testing to select candidates for nerve decompression. J Am Podiatr Med Assoc 95:433-437, 2005

27. Mallouhi A, Pülzl P, Trieb T, Piza H, Bodner G: Predictors of carpal tunnel syndrome: accuracy of gray-scale and color Doppler sonography. AJR Am J Roentgenol 186:1240-1245, 2006

28. Martinoli C, Bianchi S, Cohen M, Graif M: Ultrasound of peripheral nerves. J Radiol 86:1869-1878, 2005

29. Martinoli C, Bianchi S, Gandolfo N, Valle M, Simonetti S, Derchi LE: US of nerve entrapments in osteo fi brous tunnels of the upper and lower limbs. Radiographics 20:199-217, 2000

30. Martinoli C, Bianchi S, Pugliese F, Bacigalupo L, Gauglio C, Valle M, et al: Sonography of entrapment neuropathies in the upper limb (wrist excluded). J Clin Ultrasound 32:438-450, 2004

31. Nakamichi K, Tachibana S: Ultrasonographic measurement of median nerve cross-sectional area in idiopathic carpal tunnel syndrome: diagnostic accuracy. Muscle Nerve 26:798-803, 2002

32. Okamoto M, Abe M, Shirai H, Ueda N: Morphology and dynamics of the ulnar nerve in the cubital tunnel. Observation by ultrasonography. J Hand Surg [Br] 25:85-89, 2000

33. Peer S, Bodner G, Meirer R, Willeit J, Piza-Katzer H: Examination of postoperative peripheral nerve lesions with highresolution sonography. AJR Am J Roentgenol 177:415-419, 2001

34. Quinn TJ, Jacobson JA, Craig JG, van Holsbeeck MT: Sonography of Morton's neuromas. AJR Am J Roentgenol 174:1723-1728, 2000

35. Shapiro RS, Wagreich J, Parsons RB, Stancato-Pasic A, Yeh HC, Lao R: Tissue harmonic imaging sonography: evaluation of image quality compared with conventional sonography. AJR Am J Roentgenol 171:1203-1206, 1998
36. Shattuck DP, von Ramm OT: Compound scanning with a phased array. Ultrason Imaging 4:93-107, 1982

37. Silvestri E, Martinoli C, Derchi LE, Bertolotto M, Chiaramondia M, Rosenberg I: Echotexture of peripheral nerves: correlation between US and histologic findings and criteria to differentiate tendons. Radiology 197:291-296, 1995

38. Solbiati L, De Pra L, Ierace T, Bellotti E, Denchi LE: Highresolution sonography of the recurrent laryngeal nerve: anatomic and pathologic considerations. AJR Am J Roentgenol 145:989-993, 1985

39. Sugimoto H, Miyaji N, Ohsawa T: Carpal tunnel syndrome: evaluation of median nerve circulation with dynamic contrastenhanced MR imaging. Radiology 190:459-466, 1994

40. Thoirs K, Williams M, Phillips M: Ultrasonographic measurements of the ulnar nerve at the elbow. Role of confounders. J Ultrasound Med 27:737-743, 2008

41. Walker FO: Imaging nerve and muscle with ultrasound. Suppl Clin Neurophysiol 57:243-254, 2004

42. Werner RA, Jacobson JA, Jamadar DA: Influence of body mass index on median nerve function, carpal tunnel pressure, and cross-sectional area of the median nerve. Muscle Nerve 30:481-485, 2004

43. Wong SM, Griffith JF, Hui ACF, Lo SK, Fu M, Wong KS: Carpal tunnel syndrome: diagnostic usefulness of sonography. Radiology 232:93-99, 2004

44. Yoon JS, Walker FO, Cartwright MS: Ultrasonographic swelling ratio in the diagnosis of ulnar neuropathy at the elbow. Muscle Nerve 38:1231-1235, 2008

45. Ziswiler HR, Reichenbach S, Vögelin E, Bachmann LM, Villinger PM, Jüni P: Diagnostic value of sonography in patients with suspected carpal tunnel syndrome: a prospective study. Arthritis Rheum 52:304-311, 2005

Manuscript submitted October 15, 2008.

Accepted November 13, 2008.

Address correspondence to: Ralph W. Koenig, M.D., Department of Neurosurgery, University of Ulm, L-Heilmeyer-Str. 289312 Guenzburg, Germany. email: ralph.koenig@uni-ulm.de. 